\title{
LA HISTORIA NATURAL DEL ACLARAMIENTO DE ESPERMATOZOIDES EN EL SEMEN TRAS LA VASECTOMÍA
}

\author{
J.M. GÓMEZ DE VICENTE, I. ROMERO CAGIGAL, C. BLANCO*, J. PASTOR*, \\ A. MORENO SANTURINO, D. SANTOS ARRONTES, E. MIRAVALLES*, \\ A. BERENGUER SÁNCHEZ
}

Departamentos de Urología y *Bioquímica. Hospital Universitario de Getafe. Madrid.

Actas Urol Esp. 28 (4): 286-289, 2004

\section{RESUMEN}

LA HISTORIA NATURAL DEL ACLARAMIENTO DE ESPERMATOZOIDES

EN EL SEMEN TRAS LA VASECTOMÍA

OBJETIVO: Estudiar el tiempo necesario para obtener un seminograma negativo tras la vasectomía.

MATERIAL Y MÉTODO: Revisamos 239 vasectomías consecutivas durante un año. Todas ellas fueron realizadas de forma ambulatoria. El tiempo de seguimiento fue de 41-853 días (media 144, mediana 104). El primer seminograma fue solicitado entre 1 y 6 meses tras la intervención. A los pacientes que presentaron espermatozoides en la primera muestra de semen, se les solicitó una nueva muestra cada dos meses hasta que el seminograma fuese negativo. La probabilidad de azoospermia fue estudiada mediante curvas de Kaplan-Meier.

RESULTADOS: Al final del periodo de seguimiento, 31 pacientes (13\%) seguían teniendo espermatozoides en el seminograma. A pesar de ello, 10 pacientes (4,2\%) dejaron de acudir a la consulta. El tiempo requerido para obtener un seminograma negativo osciló entre 58 y 362 días (media 133, mediana 99). La probabilidad de quedar azoospérmico a los 200 y 260 días tras la vasectomía, fue del 80 y $90 \%$ respectivamente. Se realizaron un total de 328 seminogramas (rango 1-4, media 1,37, mediana 1).

CONCLUSIONES: Se necesita un mínimo de 200 dias (6,6 meses) para que el 80\% de nuestros pacientes queden azoospérmicos. Solicitar el primer seminograma 7 meses tras la vasectomía es rentable, reduciendo el número de visitas médicas innecesarias e incrementando la rentabilidad de esta prueba.

PALABRAS CLAVE: Vasectomía. Seminograma. Azoospermia.

\section{ABSTRACT}

THE NATURAL HISTORY OF SPERM CLEAREANCE AFTER VASECTOMY

OBJECTIVE: To study the time required to obtain a negative sperm analysis after vasectomy.

MATERIAL AND METHODS: We reviewed 239 consecutive vasectomies performed between september 1998 and september 1999. All of them were done in an ambulatory basis. Follow up interval was 41-853 days (mean 144, median 104). The first semen analysis was requested between 1 and 6 months after the surgical procedure. If the sample still showed spermatozoa, then a new one was requested every two months. Probability of becoming azoospermic was studied with Kaplan-Meier curves.

RESULTS: Persistent spermatozoa could be found in 31 patients (13\%) at the end of follow-up. Despite having a positive semen analysis, 10 patients $(4.2 \%)$ discontinued medical visits. Time required to obtain a negative sperm count ranged from 58 to 362 days (mean 133, median 99). The probability of being azoospermic 200 and 260 days after vasectomy was 80-90\% respectively. A total of 328 semen analysis were requested (range 1-4, mean 1.37, median 1)

CONCLUSIONS: A minimum of 200 days (6.6 months) are needed to clear all the spermatozoa in semen after vasectomy in $80 \%$ of our patients. Requesting the first semen sample 7 months after vasectomy is cost-effective, reducing unnecesary medical visits and increasing the rentability of this test.

Key words: Vasectomy. Semen analysis. Azoospermia. 
$\mathrm{L}$ a vasectomía se ha convertido en un procedimiento habitual para la contracepción masculina. Existe controversia sobre el tiempo necesario para obtener un seminograma negativo tras la vasectomía ${ }^{1-5}$. Schmidt afirma, que para alcanzar la azoospermia es más importante el número de eyaculaciones que el tiempo transcurrido desde la vasectomía. Concluye que tras 12 a 15 eyaculaciones, el 90\% de sus pacientes queda azoospérmico ${ }^{4}$. En consecuencia, este periodo depende de las costumbres sexuales individuales.

Con frecuencia, un seminograma solicitado 3 meses después de la vasectomía (periodo probablemente suficiente para eyacular 15 veces) es informado como "espermatozoides inmóviles aislados". A algunos pacientes se les da un "alta especial" cuando esta situación persiste más de 7 meses ${ }^{11}$. Algunos autores estiman que el riesgo de embarazo en esta situación es menor que el riesgo establecido de recanalización tardía ${ }^{2}$. Sin embargo, no podemos despreciar las implicaciones médico-legales derivadas de dar el alta a un paciente con espermatozoides en el semen ${ }^{6-10}$. En este trabajo, hemos estudiado de forma retrospectiva la probabilidad de quedar azoospérmico tras una vasectomía. La finalidad es disminuir el número de visitas médicas y seminogramas innecesarios.

\section{MATERIALES Y MÉTODOS}

Revisamos 239 vasectomías realizadas de forma consecutiva en nuestro centro entre septiembre de 1998 y septiembre de 1999. Todas las intervenciones se realizaron de forma ambulatoria por diferentes cirujanos. Aunque existen ciertas variaciones en la técnica de cada uno, siempre se extirpa un segmento de conducto deferente y los cabos se coagulan y se ligan. En algunos casos también se entierra el cabo proximal en un plano quirúrgico diferente.

El primer seminograma se solicitó entre 1 y 6 meses tras la vasectomía, sin embargo, la mayor parte de ellos fue solicitado a los 3 meses. En los pacientes con persistencia de espermatozoides en la muestra, se realizó un nuevo seminograma cada 2 meses hasta lograr la azoospermia, momento en el que el paciente era dado de alta.
El semen se analiza en primer lugar mediante microscopía óptica. Una vez homogenizada la muestra, se coloca sobre un porta y se visualizan un mínimo de 100 campos a 40x. En caso de existir espermatozoides, el laboratorio nos informa sobre el número de espermatozoides por campo de gran aumento y su motilidad. En caso de existir menos de 1.000 espermatozoides de escasa vitalidad por cada 100 campos de gran aumento, se nos informa de la existencia de "espermatozoides inmóviles aislados”. Cuando no se observan espermatozoides en la muestra, ésta es centrifugada a 3000 rpm durante 5 minutos, y el sedimento se observa al microscopio de la forma antes descrita.

Se utilizaron curvas de Kaplan-Meier para el evento azoospermia. El análisis estadístico se realizó mediante el uso del programa estadístico SPSS (SPSS para Windows, versión 8.0.0 Copyright@ SPSS Inc., Chicago IL, USA).

\section{RESULTADOS}

Todos los pacientes fueron controlados en consulta al menos una vez. Diez pacientes $(4,2 \%)$ dejaron de acudir a consulta a pesar de no haber negativizado el seminograma y fueron excluidos del análisis estadístico.

De los restantes 229 pacientes, 21 (9,1\%) presentaban "espermatozoides inmóviles aislados" en su última revisión. El tiempo de seguimiento fue de 41-853 días (media 144, DE 86, mediana 104). Los restantes 208 pacientes quedaron azoospérmicos durante el seguimiento. El tiempo para negativizar el seminograma fue de 58-362 días (media 133, DE 62, mediana 99).

La probabilidad de quedar azoospérmico a lo largo del tiempo es mostrada en la Figura 1. La probabilidad de quedar azoospérmico a los 3, 6 , 9 y 12 meses es de 23,9\%, 70,6\%, 90,5\% y 98,3\% respectivamente (Tabla I).

Se solicitaron un total de 328 seminogramas (rango 1-4, media 1,3, DE 0,6, mediana 1).

\section{DISCUSIÓN}

Un paciente sometido a una vasectomía es dado de alta en el momento en que queda azoospérmico. Naturalmente, la satisfacción del paciente es tanto mayor cuanto más precozmente se alcance dicho estado. Tras estudiar 6.248 vasectomías, Schmidt concluye que tras 


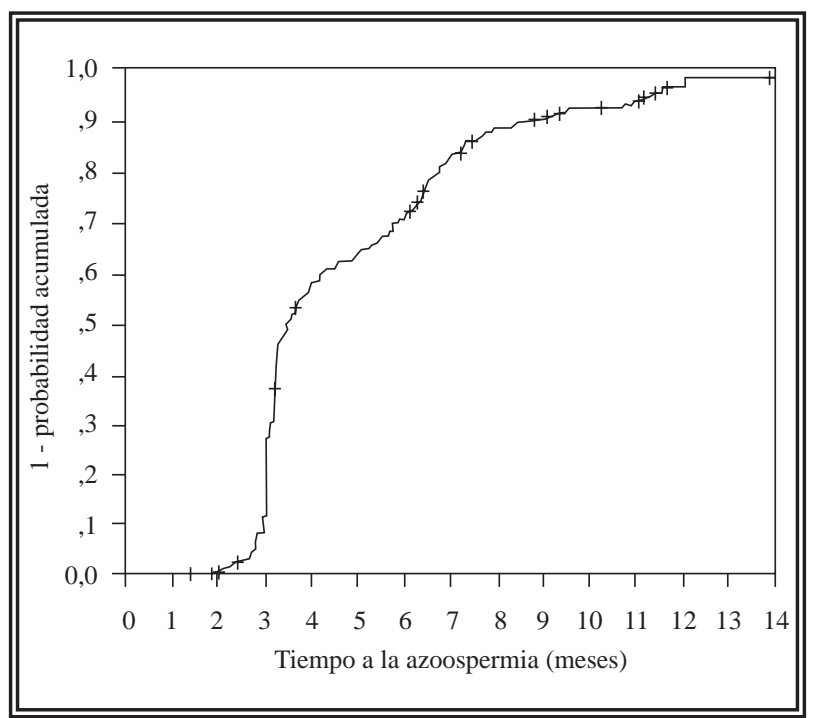

FIGURA 1. Probabilidad de quedar azoospérmico a los largo del tiempo.

\section{TABLA I}

PROBABILIDAD ACUMULADA DE AZOOSPERMIA EXPRESADA EN MESES

\begin{tabular}{|c|c|c|}
\hline $\begin{array}{c}\text { Meses tras la } \\
\text { vasectomia }\end{array}$ & $\begin{array}{c}\text { Probabilidad de } \\
\text { azoospermia (\%) }\end{array}$ & $\begin{array}{c}\text { Eventos } \\
\text { acumulados }\end{array}$ \\
\hline 2 & 0,88 & 2 \\
\hline 3 & 23,9 & 54 \\
\hline 4 & 57,9 & 130 \\
\hline 5 & 68,4 & 143 \\
\hline 6 & 70,6 & 158 \\
\hline 7 & $\mathbf{8 3 , 3}$ & $\mathbf{1 8 5}$ \\
\hline 8 & 88,4 & 195 \\
\hline 10 & 90,5 & 202 \\
\hline 11 & 92,4 & 204 \\
\hline 12 & 93,7 & 208 \\
\hline
\end{tabular}

15 eyaculaciones, el $90 \%$ de sus pacientes queda azoospérmico ${ }^{4}$. Tras la publicación de este artículo, se instauró de forma generalizada la recomendación de solicitar el primer seminograma 6 semanas después de la intervención ${ }^{8}$. Sin embargo, en este estudio, tan sólo el $23,9 \%$ de los pacientes queda azoospérmico a los 3 meses.
Por otra parte, otros autores han encontrado resultados similares a los nuestros, recomendando que transcurra un periodo de 6 meses entre la intervención y el primer seminograma ${ }^{1}$.

Sin embargo, el riesgo de embarazo cuando existen "espermatozoides inmóviles aislados" 7 meses después de la intervención es bajo. Se estima que este riesgo es menor que el de recanalización espontánea ${ }^{2,11}$.

En nuestras manos, la probabilidad de quedar azoospérmico 7 meses después de la vasectomía es del 83,3\%. En los demás pacientes quedan "espermatozoides inmóviles aislados", por lo que podrian ser dados de alta asumiendo que la probabilidad de embarazo es muy baja a pesar de no haber negativizado el seminograma.

Algunos consideran que un periodo tan largo puede contrariar al paciente ${ }^{7}$. Sin embargo, desde el final de este estudio, solicitamos el primer seminograma 7 meses después de la vasectomía y la aceptación ha sido buena. En una encuesta realizada a pacientes británicos vasectomizados en relación a la solicitud de seminogramas tras la vasectomía, el $27 \%$ prefería aportar la primera muestra a los 3 meses, el 35\% a los 6 meses y el $38 \%$ no mostraban preferencia alguna ${ }^{1}$.

Es importante destacar que algunos pacientes son fértiles a pesar de contar con escasos espermatozoides inmóviles en el seminograma ${ }^{9,10}$ y que las diferencias en el recuento de espermatozoides entre pacientes fértiles e infértiles no está claramente definida ${ }^{6}$. Existen incluso algunos casos de paternidad probada en padres azoospérmi$\cos ^{3}$. Por lo tanto, conviene que los pacientes sean informados por escrito de que existe una posibilidad remota de embarazo ${ }^{9-11}$.

Retrasar la solicitud del seminograma al $7^{\circ}$ mes podría mejorar la existencia de nuestras consultas, así como reducir la posibilidad de problemas legales.

\section{CONCLUSIONES}

En nuestra experiencia, la probabilidad de quedar azoospérmico 7 meses después de la vasectomía es de $83,3 \%$. Solicitar el primer seminograma tras este periodo repercute en una menor presión asistencial y en una mayor rentabilidad de esta prueba. 


\section{REFERENCIAS}

1. SMITH AG, CROOKS J, SINGH NP, SCOTT R, LLOYD SN.: Is the timing of post-vasectomy seminal analysis important?. Br J Urol 1998; 81: 458-460.

2. BENGER JR, SWAMI SK, GINGELL JC.: Persistent spermatozoa after vasectomy: a survey of british urologists. Br J Urol 1995; 76: 376-379.

3. O`BRIEN TS, CRANSTON D, ASHWIN P, TURNER E, MACKENZIE IZ, GUILLEBAUD J.: Temporary reappearance of sperm 12 months after vasectomy clearance. Br J Urol 1995; 76: 371-372.

4. SCHMIDT SS.: Vasectomy by section, luminal fulguration and fascial interposition: results from 6248 cases. Br J Urol 1995; 76: 373-375.

5. SMITH JC, CRANSTON D, O`BRIEN T, GUILLEBAUD J, HINDMARSH.: Fatherhood without apparent spermatozoa after vasectomy. Lancet 1994; 344: 30.

6. GUZICK DS, OVERSTREET JW, FACTOR-LITVAK P, BRAZIL CK, NAKAJIMA ST, COUTIFARIS C, CARSON SA, CISNEROS P, STEINKAMPF MP, KILL JA, XU D, PHIL M, VOGEL DL.: Sperm morphology, motility, and concentration in fertile and infertile men. $N$ Engl J Med 2001; 345 (19), nov 8: 1388-1393.

7. SCHMIDT SS.: Is the timing of post-vasectomy analysis important? Correspondence. $\mathrm{Br} J$ Urol 1998 sep; 82 (3): 461.
8. SCHLEGEL PN, GOLDSTEIN M.: Vasectomy. AUA update series. Lesson 13, volume XI.

9. THOMPSON JA, LINCONLN PJ, MORTIMER P.: Paternity by a seemingly infertile vasectomized man. Br Med $J$ 1993; 307: 299-300.

10. BELKER AM, SEXTER MS, SWEITZER SJ, RAFF MJ.: The high rate of noncompliance for postvasectomy semen examination: medical and legal considerations. J Urol 1990 aug; 144 (2 Pt 1): 284286.

11. DAVIES AH, SHARP RJ, CRANSTON D, MITCHELL RG.: The long-term outcome following "special clearance” after vasectomy. Br J Urol 1990 aug; 66 (2): 211-212.

Dr. J.M. Gómez de Vicente

(Servicio de Urología)

Hospital Univ. de Getafe

Ctra. de Toledo km. 12,500

28905 Getafe (Madrid)

(Trabajo recibido el 24 marzo de 2003) 